Sādhanā Vol. 29, Part 6, December 2004, pp. 617-628. ( ) Printed in India

\title{
Hydrodynamic coefficients for water-wave diffraction by spherical structures
}

\author{
SWAROOP NANDAN BORA \\ Department of Mathematics, Indian Institute of Technology, Guwahati 781 039, \\ India \\ e-mail: swaroop@iitg.ernet.in
}

MS received 10 June 2004; revised 7 September 2004

\begin{abstract}
Evaluation of hydrodynamic coefficients and loads on submerged or floating bodies is of great significance in designing these structures. Some special regular-shaped geometries such as those of cylindrical (circular, elliptic) and spherical (hemisphere, sphere, spheroid) structures are usually considered to obtain analytical solutions to wave diffraction and radiation problems. The work presented here is the result of water-wave interaction with submerged spheres. Analytical expressions for various hydrodynamic coefficients and loads due to the diffraction of water waves by a submerged sphere are obtained. The exciting force components due to surge and heave motions are derived by solving the diffraction problem. Theory of multipole expansions is used to express the velocity potentials in terms of an infinite series of associated Legendre polynomials with unknown coefficients and the orthogonality of the polynomials is utilized to simplify the expressions. Since the infinite series appearing in various expressions have excellent truncation properties, they are evaluated by considering only a finite number of terms. Gaussian quadrature is used to evaluate the integrals. Numerical estimates for the analytical expressions for the hydrodynamic coefficients and loads are presented for various depth to radius ratios. Consideration of more values for depth makes it easy to compare the results with those available. The results obtained match closely with those obtained earlier by Wang and $\mathrm{Wu}$ and their coworkers.
\end{abstract}

Keywords. Diffraction; Legendre polynomial; surge and heave motions; exciting force.

\section{Introduction}

Since the days of Havelock, the study of water waves has been considered a major part of fluid dynamics. The forces exerted by the surface waves on a structure in the water are very important for designing these structures. Accurate prediction of wave loads becomes indispensable in order to design safe structures.

When the size of the structure is comparable to the dominant wavelength, or when the structure is large enough to span a significant fraction of a wavelength, the incoming waves 
undergo significant diffraction or scattering upon arriving at the structure. The presence of the structure alters the conditions of the wave field surrounding it. Hence the diffraction of the waves is a major factor which cannot be ignored. That compels us to take into account the diffraction of the waves, from the surface of the structure, in evaluating the wave forces. This is generally known as the diffraction theory. Adopting this method, analytical solution in closed forms are possible for a number of structures.

A rigid floating or submerged structure may undergo six degrees of freedom: three translational and three rotational. Assuming a suitable coordinate system, $O X Y Z$, the translational motions are in the $x, y$ and $z$ directions (here longitudinal along $x$, transverse along $y$ and vertical along $z$ ) which are referred to as surge, sway and heave respectively. The rotational motions about the $x, y$ and $z$ directions are referred to as roll, pitch and yaw respectively. Here, the $z$-axis is considered to be vertically downwards from the still water level. Often the structure is constrained to have fewer than six degrees of freedom, for example, the type of mechanical connection used to fasten the structure to the sea-floor. Physically, vertical and longitudinal motions are of primary importance for a floating or submerged body.

Havelock can be considered the pioneer in the area of hydrodynamic loading on spherical structures. He calculated the wave resistance of a submerged spheroid by replacing it with a distribution of sources and sinks, or of doublets, using the linearized free surface condition (Havelock 1931). Much later, he also discussed the fluid motion due to a half-immersed floating sphere undergoing small heaving oscillations. He obtained the velocity potential as a series, with the unknown coefficients given by an infinite set of equations (Havelock 1955).

Hulme considered the heave and surge motions of a floating hemisphere to derive addedmass and damping coefficients associated with periodic motions. He also briefly discussed the derivation of the long- and short-wave asymptotics of these coefficients. This method can also be used to treat the physically distinct, but mathematically similar, problem of the diffraction of waves by a fixed hemisphere (Hulme 1982). Considering a submerged vehicle as a neutrally buoyant sphere, Wang discussed the free motions of a submerged vehicle with a spherical hull form, but with different metacentric heights. The associated radiation and diffraction problems were solved independently, in order to examine the motions and the stability of the submerged hull form (Wang 1986). The works of Hulme and Wang were based on the multipole expansions method of Thorne (1953) which proved to be very successful for periodic motions without forward speeds. However, this method did not seem to be applicable to the problem of a body with forward speed.

$\mathrm{Wu}$ and Taylor analysed the hydrodynamic problem of a submerged spheroid in waves, based on linearized potential theory. The problem of a submerged spheroid in head or following seas was considered and the subsequent formulation was presented. It was suggested that this method could be extended to deal with the problem of oscillating bodies at forward speed (Wu \& Taylor 1987). As forward speed significantly affects the body-surface, freesurface and radiation boundary conditions imposed on the velocity potential corresponding to the oscillations of the body, $\mathrm{Wu}$ and Taylor considered a submerged sphere advancing in regular deep water waves at constant forward speed. Linearized potential theory was adopted and a distribution of sources over the surface of the sphere was expanded into a series of Legendre polynomials. Although linearized potential theory has very little physical significance for a spherical structure, the solution clarified doubts about the influence of forward speed on hydrodynamic forces (Wu \& Taylor 1988). Wu and Taylor also analysed the problem of wave radiation and diffraction by submerged spheroids, using linearized three-dimensional poten- 
tial flow theory. The solution was obtained by expanding the velocity potential into a series of Legendre polynomials in a spheroidal coordinate system. However, this solution also could not be extended for cases with forward speed (Wu \& Taylor 1989). Later, they considered a submerged sphere moving in a circular path at constant angular velocity, the analysis being based on the linearized velocity potential theory. The potential was expressed by means of a Green's function and a distribution of sources over the body surface, written in terms of Legendre polynomials (Wu \& Taylor 1990).

$\mathrm{Wu}$ (1994) considered the hydrodynamic problem of a sphere submerged below a free surface and undergoing large amplitude oscillation. Velocity potential theory was applied and the body surface boundary condition was satisfied on its instantaneous position, with linearized free-surface boundary conditions. Wu et al (1994) presented a solution for the wave induced drift forces acting on a submerged sphere in finite water depth, based on linearized potential theory. The theory of multipole expansions was used in terms of an infinite series of Legendre polynomials with unknown coefficients. The series expression for the second order mean forces (drift forces) was provided by integrating the fluid pressure over the body surface. The horizontal drift force was also expressed by a series solution, obtained by using the farfield method (Wu \& Taylor 1990). The method of multipole expansions used in the problem solution gave rise to some problems due to non-consideration of more values of water depth and body submergence values. Bora (2002) discussed the diffraction and radiation problem of a submerged sphere in finite water depth by breaking the problem separately considering the diffraction and radiation parts. However, that analytical solution could not show how close the results could be with the available accepted results.

\section{Mathematical formulation}

We assume that the fluid flow is homogeneous, inviscid, incompressible and irrotational. The waves are also assumed to be of small amplitude. We consider the coefficients related to the motion with three degrees of freedom, namely, two translational motions in the $x$ and $z$ directions, i.e. surge and heave motions respectively, and the rotational motion about the $y$ direction, i.e. pitch motion. We consider a surface wave of amplitude $A$ and angular frequency $\sigma$ incident on a sphere of radius $a$ submerged in water of finite depth $d$. The wave is parallel to the $x$-axis at the time of incidence on the sphere and propagates along the positive direction.

We consider two sets of coordinate systems. One is a right-handed Cartesian coordinate system $(x, y, z)$, in which the $x-y$ plane coincides with the undisturbed free surface and the $z$-axis is taken vertically downwards from the still water level. The other coordinate system is the spherical coordinate system $(r, \theta, \psi)$ with the origin at the geometric centre of the sphere. Figure 1 shows the axes systems with a sphere of radius $a$ in water of depth $d$ with its geometric centre located at $(0,0, h)$ with respect to the Cartesian coordinate system.

The relationship between the coordinate systems is:

$$
\begin{aligned}
R & =\left(x^{2}+y^{2}\right)^{1 / 2}, \\
r & =\left[R^{2}+(z-h)^{2}\right]^{1 / 2}, \\
\tan \theta & =R /(z-h), \quad \text { for } \quad 0 \leq \theta \leq \pi, \\
\tan \psi & =y / x, \quad \text { for } \quad-\pi \leq \psi \leq \pi .
\end{aligned}
$$




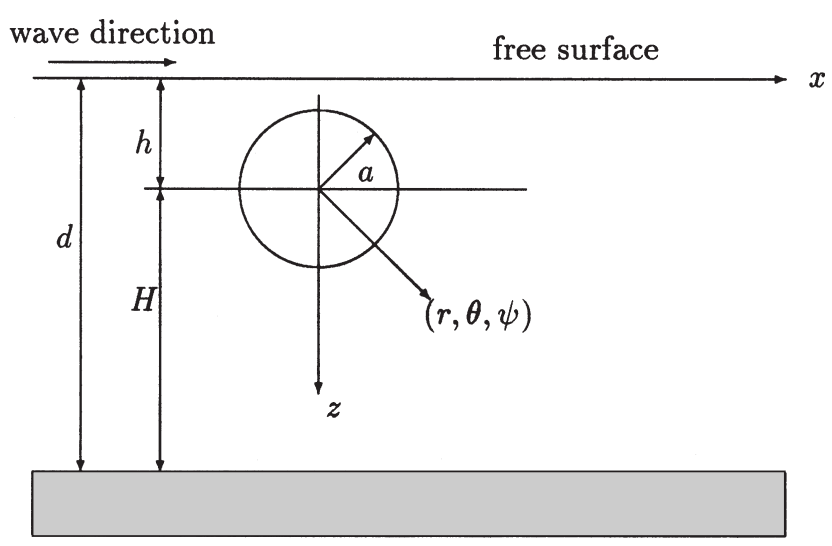

sea bed
Figure 1. Reference coordinate system.

For incompressible and inviscid flow, and for small amplitude wave theory with irrotational motion, we can express the fluid motion by introducing a velocity potential $\Phi(r, \theta, \psi, t)$. This $\Phi$ can be written as:

$$
\Phi(r, \theta, \psi, t)=\operatorname{Re}[\phi(r, \theta, \psi) \exp (-i \sigma t)]
$$

where Re stands for the real part.

The motion is assumed harmonic. Also, from Bernoulli's equation, we get pressure, $P(r, \theta, \psi, t)$ as

$$
P=-\rho(\partial \Phi / \partial t) .
$$

The $t$-independent potential $\phi$ can be written as $\phi=\phi_{I}+\phi_{D}$.

To obtain the velocity potential $\phi$, the following boundary problem must be solved.

(1) Laplace's equation in spherical coordinates:

$$
\nabla^{2} \phi=0
$$

(2) Free surface condition:

$$
\partial \phi / \partial z+K \phi=0, \quad \text { on } \quad z=0 .
$$

(3) Bottom boundary condition:

$$
\partial \phi / \partial z=0, \quad z=d .
$$

(4) Radiation condition:

$$
\lim _{R \rightarrow \infty} \sqrt{R}\left(\frac{\partial}{\partial R}-i k_{0}\right) \phi=0
$$

where $K=\sigma^{2} / g$ and $k_{0}$ is the finite depth wave number defined by

$$
k_{0} \sinh k_{0} d-K \cosh k_{0} d=0,
$$


and the incident and diffraction potentials satisfy the body surface condition

$$
\partial \phi_{I} / \partial \mathbf{n}=-\partial \phi_{D} / \partial \mathbf{n}, \quad \text { on } \quad r=a,
$$

where $\mathbf{n}$ denotes the normal vector from the body surface to the fluid.

\section{Incident potential}

Incoming waves of amplitude $A$ and frequency $\sigma$, propagating in the positive $x$-direction, can be described by the following incident velocity potential,

$$
\phi_{I}=\frac{A g}{\sigma} \frac{\cosh k_{0}(z-d)}{\cosh k_{0} d} \exp \left(i k_{0} R \cos \psi\right) .
$$

Using the relation between exponential function and Bessel function (McLachlan 1941), this may be expressed as,

$$
\phi_{I}=\frac{A g}{\sigma} \frac{\cosh k_{0}(z-d)}{\cosh k_{0} d} \sum_{m=0}^{\infty} \epsilon_{m} i^{m} J_{m}\left(k_{0} R\right) \cos m \psi,
$$

where $\epsilon_{0}=1$ and $\epsilon_{m}=2$ for $m \geq 1$.

Using multiple expansions (Thorne 1953), the incident potential can be expressed in terms of associated Legendre's polynomial as:

$$
\phi_{I}(r, \theta, \psi)=\sum_{m=0}^{\infty} \tilde{\phi}_{I}(r, \theta) \cos m \psi,
$$

where

$$
\begin{aligned}
\tilde{\phi}_{I}= & \frac{A g \epsilon_{m} i^{m}}{2 \sigma \cosh k_{0} d} \sum_{s=m}^{\infty}\left\{(-1)^{s+m} \exp \left(k_{0}(d-h)\right)\right. \\
& \left.+\exp \left(k_{0}(h-d)\right)\right\} \frac{\left(k_{0} r\right)^{s}}{(s+m) !} P_{s}^{m}(\cos \theta) .
\end{aligned}
$$

Changing $s$ to $s+m$, we can write $\tilde{\phi}_{I}(r, \theta)$ as

$$
\tilde{\phi}_{I}(r, \theta)=\frac{A g}{\sigma} \epsilon_{m} i^{m} \sum_{s=0}^{\infty} \chi_{s} \frac{\left(k_{0} r\right)^{s+m}}{(s+2 m) !} P_{s+m}^{m}(\cos \theta),
$$

where

$$
\begin{aligned}
\chi_{s}= & \frac{(-1)^{s} \exp \left(k_{0}(d-h)\right)+\exp \left(-k_{0}(d-h)\right)}{2 \cosh k_{0} d}, \\
& = \begin{cases}\frac{\cosh k_{0}(d-h)}{\cosh k_{0} d}, & s=0,2,4,6, \ldots \\
-\frac{\sinh k_{0}(d-h)}{\cosh k_{0} d}, & s=1,3,5, \ldots\end{cases}
\end{aligned}
$$

Hence, the incident potential $\phi_{I}$ can be written in the final form as

$$
\phi_{I}(r, \theta, \psi)=\sum_{m=0}^{\infty} \frac{A g}{\sigma} \epsilon_{m} i^{m} \sum_{s=0}^{\infty} \chi_{s} \frac{\left(k_{0} r\right)^{s+m}}{(s+2 m) !} P_{s+m}^{m}(\cos \theta) \cos m \psi .
$$




\section{Diffraction potential}

The diffraction velocity potential $\phi_{D}$ satisfies (3)-(6) and (8). We can express this potential by making it $\psi$-independent as:

$$
\phi_{D}(r, \theta, \psi)=\sum_{m=0}^{\infty} \tilde{\phi}_{D}(r, \theta) \cos m \psi,
$$

where the $\psi$-independent potential is

$$
\tilde{\phi}_{D}(r, \theta)=\sum_{n=m}^{\infty} a^{n+2} A_{m n} G_{n}^{m} .
$$

Here, $A_{m n}$ are the unknown complex coefficients and $G_{n}^{m}(r, \theta, z)$ are the multipole potentials. Multipole potentials are solutions of Laplace's equation which satisfy the free surface and bottom boundary conditions and behave like outgoing waves from the singular point which, in this case, is the centre of the sphere. We can express $G_{n}^{m}$ as,

$$
\begin{aligned}
G_{n}^{m}= & \frac{P_{n}^{m}(\cos \theta)}{r^{n+1}}+\frac{P_{n}^{m}(\cos \alpha)}{r_{1}^{n+1}}+\frac{1}{(n-m) !} \int_{0}^{\infty} k^{n} \cosh k(z-d) J_{m}(k R) \\
& \times \frac{(K+k)\left[\exp (-k(d+H))+(-1)^{n+m} \exp (-k h)\right]}{k \sinh k d-K \cosh k d} \mathrm{~d} k,
\end{aligned}
$$

The quantities $\alpha$ and $r_{1}$ are defined as:

$$
\begin{aligned}
r_{1} & =\left(R^{2}+(d+H-z)^{2}\right)^{1 / 2} \\
\tan \alpha & =R /(d+H-z),
\end{aligned}
$$

where $R, d$ and $H$ have already been defined.

The line integration in the expression for $G_{n}^{m}$ passes under the singular point of the integrand at $k=k_{0}$. The potentials $G_{n}^{m}$ and $\phi_{D}$ satisfy Laplace's equation, the free surface condition, the bottom surface condition and the radiation condition.

The second and third terms in (17) can be expanded, in the region near the body surface, into a series of associated Legendre's polynomials by

$$
\frac{P_{n}^{m}(\cos \alpha)}{r_{1}^{n+1}}=\sum_{s=0}^{\infty} B_{n s}^{m}\left(\frac{r}{2 H}\right)^{s+m} P_{s+m}^{m}(\cos \theta)
$$

and

$$
\begin{aligned}
& \int_{0}^{\infty} \frac{(K+k)\left[\exp (-k(d+H))+(-1)^{n+m} \exp (-k h)\right]}{(n-m) !(k \sinh k d-K \cosh k d)} \\
& k^{n} \cosh k(z-d) J_{m}(k R) \mathrm{d} k=\sum_{s=0}^{\infty} C_{s}(n, m)\left(\frac{r}{2 H}\right)^{s+m} P_{s+m}^{m}(\cos \theta),
\end{aligned}
$$


where $B_{n s}^{m}$ and $C_{s}(n, m)$ are given by

$$
\begin{aligned}
B_{n s}^{m} & =\frac{1}{(2 H)^{n+1}} \frac{(s+n+m) !}{(s+2 m) !(n-m) !}, \\
C_{s}(n, m) & =\frac{(2 H)^{s+m}}{(n-m) !(s+2 m) !} \\
& \int_{0}^{\infty} \frac{(K+k)\left[\exp (-k(d+H))+(-1)^{n+m} \exp (-k h)\right]}{k \sinh k d-K \cosh k d} u_{s}(k H) \mathrm{d} k,
\end{aligned}
$$

with $u_{s}(k H)$ as

$$
u_{s}(k H)=\left\{\begin{array}{ll}
\cosh k H, & s=0,2,4, \ldots \\
-\sinh k H, & s=1,3,5, \ldots
\end{array} .\right.
$$

Hence the multipole potentials $G_{n}^{m}$ can finally be written as

$$
G_{n}^{m}=\frac{P_{n}^{m}(\cos \theta)}{r^{n+1}}+\sum_{s=0}^{\infty}\left[B_{n s}^{m}+C_{s}(n, m)\right]\left(\frac{r}{2 H}\right)^{s+m} P_{s+m}^{m}(\cos \theta) .
$$

Using the body boundary condition (8), we may write,

$$
\left.\sum_{n=m}^{\infty} a^{n+2} A_{m n} \frac{\partial G_{n}^{m}}{\partial r}\right|_{r=a}=-\left.\frac{\partial \tilde{\phi}_{I}}{\partial r}\right|_{r=a}
$$

We find $\partial G_{n}^{m} / \partial r$ and $\partial \tilde{\phi}_{I} / \partial r$ from the expressions for $G_{n}^{m}$ and $\tilde{\phi}_{I}$ from eqns (23) and (12) respectively and evaluate their values at $r=a$. Multiplying the result by $P_{n}^{m}(\cos \theta) \sin \theta$ and integrating with respect to $\theta$ in $0 \leq \theta \leq \pi$ and using the orthogonality property of associated Legendre's polynomials, we obtain

$$
\begin{aligned}
\sum_{n=m}^{\infty} A_{m n}\left[-(n+1) \delta_{n s}\right. & \left.+\left(B_{n s}^{m}+C_{s}(n, m)\right)\left(\frac{a}{2 H}\right)^{s+m}(s+m) a^{n+1}\right] \\
& =-\frac{A g}{\sigma} \epsilon_{m} i^{m} \chi_{s} \frac{\left(k_{0} a\right)^{s+m}}{(s+2 m) !}(s+m) a^{-1},
\end{aligned}
$$

which in compact form gives rise to

$$
\sum_{n=m}^{\infty} A_{m n} E_{n s}^{m}=T_{s}^{m} \quad \text { for } \quad s=m, m+1, m+2, \ldots,
$$

where

$$
\begin{aligned}
T_{s}^{m} & =-\frac{A g k_{0}}{\sigma} \epsilon_{m} i^{m}\left(k_{0} a\right)^{s-1} \frac{s}{(s+m) !} \chi_{s-m}, \\
E_{n s}^{m} & =-(n+1) \delta_{n s}+D_{n}^{m}(s-m), \\
D_{n}^{m}(s) & =a^{n+1}(s+m)\left(\frac{a}{2 H}\right)^{s+m}\left[C_{s}(n, m)+B_{n s}^{m}\right] .
\end{aligned}
$$


The diffraction potential $\phi_{D}$ has the final form

$$
\begin{aligned}
\phi_{D}= & \sum_{m=0}^{\infty} \sum_{n=m}^{\infty}\left[\frac{P_{n}^{m}(\cos \theta)}{r^{n+1}}+\sum_{s=0}^{\infty}\left\{B_{n s}^{m}+C_{s}(n, m)\right\}\left(\frac{r}{2 H}\right)^{s+m} P_{s+m}^{m}(\cos \theta)\right] . \\
& \times A_{m n} a^{n+2} \cos m \psi
\end{aligned}
$$

Equation (25) is a complex matrix equation in the unknowns $A_{m n}$. Since the infinite series appearing in (26) and (28) have excellent truncation properties, the infinite matrices can be truncated, after a finite number of terms, and we solve (25) numerically. Commercially available complex matrix inversion routines are used to obtain the solution of the modified equation. Once these coefficients are known, the diffraction problem is completely known.

\subsection{Exciting forces}

The forces associated with the incident and diffraction potentials are the exciting forces which play a very important role in the wave field for a structure in water. The exciting forces $F_{j}^{(e)}$ can be obtained from:

$$
F_{j}^{(e)}=\left.2 i \rho a^{2} \sigma A \int_{0}^{\pi} \int_{0}^{\pi} \phi_{I D}\right|_{r=a} n_{j} \sin \theta \mathrm{d} \theta \mathrm{d} \psi,
$$

where $j=0$ corresponds to heave motion and $j=1$ corresponds to surge motion and we have written $\phi_{I D}=\phi_{I}+\phi_{D}$, where

$$
n_{j}=-P_{1}^{j}(\cos \theta) \cos j \psi, \quad j=0,1 .
$$

Using the body surface boundary condition (8), $\chi_{s}$ can be determined as

$$
\begin{aligned}
\chi_{s}= & -\frac{\sigma a(s+2 m) !}{A g \epsilon_{m} i^{m}\left(k_{0} a\right)^{s+m}(s+m)} \\
& \times \sum_{n=m}^{\infty} A_{m n}\left[-(n+1)+\left(B_{n s}^{m}+C_{s}\right)(s+m)\left(\frac{a}{2 H}\right)^{s+m} a^{n+1}\right] .
\end{aligned}
$$

Equations (14) and (29) help us in determining the value of $\phi_{I D}$ at $r=a$ :

$$
\left.\phi_{I D}\right|_{r=a}=a \sum_{m=0}^{\infty} \sum_{n=m}^{\infty}[(2 n+1) / n] A_{m n} P_{n}^{m}(\cos \theta) \cos m \psi
$$

Therefore, the exciting forces are given by:

$$
\begin{aligned}
F_{j}^{(e)} & =-\left.2 \rho \sigma a^{2} A \int_{0}^{\pi} \int_{0}^{\pi} \phi_{I D}\right|_{r=a} P_{1}^{j}(\cos \theta) \cos j \psi \cos \psi \sin \theta \mathrm{d} \theta \mathrm{d} \psi, \\
& =-\frac{2 \rho \sigma a^{2} A \pi}{\epsilon_{j}} \int_{0}^{\pi} \sum_{n=j}^{\infty} a \frac{2 n+1}{n} A_{j n} P_{n}^{j}(\cos \theta) \sin \theta \mathrm{d} \theta,
\end{aligned}
$$

where $\epsilon_{j}=1$ for $j=0, \epsilon_{j}=2$ for $j \geq 1$. 
Using the orthogonality property of associated Legendre's polynomials, we obtain

$$
\begin{aligned}
F_{j}^{(e)} & =-2 \rho \sigma \pi A a^{3} \epsilon_{j} 2[(1+j) ! /(1-j) !] A_{j 1} \\
& =-4 \rho \sigma \pi a^{3} A A_{j 1},
\end{aligned}
$$

since the terms $\epsilon_{j}$ and $\frac{(1+j) !}{(1-j) !}$ cancel out for the respective values of $j$.

Hence the surge exciting force $F_{x}^{(e)}=f_{x d}$ is given by

$$
f_{x d}=-4 \rho \sigma \pi A a^{3} A_{11}
$$

and the heave exciting force $F_{z}^{(e)}=f_{z d}$ is given by

$$
f_{z d}=-4 \rho \sigma \pi A a^{3} A_{01}
$$

\section{Results and discussions}

Now we present numerical results for the analytical expressions for various hydrodynamic coefficients and loadings derived in the last section. The complex matrix equation (25) has to be solved in order to determine the unknown coefficients $A_{m n}$ for $m=0$ and $m=1$. To compute the surge (horizontal) exciting force, $f_{x d}$, we need to solve (36). The heave (vertical) exciting force, $f_{z d}$, is evaluated by solving (37). The infinite system of equations represented by (25) is truncated and then solved numerically:

$$
\sum_{n=0}^{N_{p}} A_{m n} E_{n s}^{m}=T_{s}^{m},
$$

where $E_{n s}^{m}$ and $T_{s}^{m}$ are given by the set of equations (26)-(28).

These system of equations are solved by using a complex matrix inversion subroutine from IMSL. We select $N_{p}=20$ for our computations. We have observed throughout our numerical calculations that addition of more terms beyond 20 terms does not have any significant effect. Once $A_{01}, A_{11}$ are known, we can compute the exciting forces due to surge and heave motions.

Tables 1 to 4 give us the exciting force coefficients for both fixed submergence and fixed depth. Tables 1 and 2 respectively present surge and heave exciting forces $f_{x d}$ and $f_{z d}$ at a fixed submergence $(h / a=1.25)$ for a range of water waves $(d / a)$. Also included are the results of Wang (1986) for infinite depth and Wu et al (1994) for finite depth. There seems to be good agreement of our result with those sets of results within three significant figures although different expansions of the velocity potential have been used. It is evident that as the depth increases the surge forces also increase, the maximum variation for the values of $K a$ being between $0 \cdot 1$ and $0 \cdot 5$. The converse is true for the heave exciting forces which at this submergence reduce significantly from that in deep water. Tables 3 and 4 respectively present surge and heave exciting forces on the sphere in fixed water depth $(d / a=6 \cdot 0)$ for a range of submergence values. The force coefficients decrease significantly for increased submergence values. 
Table 1. Surge exciting forces $f_{x d}$ for $(h / a=1 \cdot 25)$.

\begin{tabular}{lcccccc}
\hline & \multicolumn{7}{c}{$d / a$} \\
\cline { 2 - 7 }$K a$ & 2.5 & 3.0 & 5.0 & $11 \cdot 0$ & $20.0^{*}$ & Wu \\
\hline $0 \cdot 10$ & 3.1539 & 2.7864 & $2 \cdot 1872$ & 1.5893 & 1.4897 & 1.4896 \\
$0 \cdot 20$ & 2.1152 & 2.1152 & 1.5902 & 1.3151 & 1.2621 & 1.2618 \\
$0 \cdot 30$ & 1.6347 & 1.3976 & 1.1861 & 1.1361 & 1.1102 & 1.1108 \\
$0 \cdot 40$ & 1.2862 & 1.1471 & 0.9861 & 0.9858 & 0.9826 & 0.9831 \\
0.50 & 1.1134 & 0.9876 & 0.8862 & 0.8852 & 0.8834 & 0.8838 \\
$0 \cdot 60$ & 0.9217 & 0.8692 & 0.8682 & 0.7809 & 0.8124 & 0.8118 \\
0.70 & 0.7692 & 0.7418 & 0.7398 & 0.6947 & 0.7395 & 0.7393 \\
$0 \cdot 80$ & 0.6824 & 0.6675 & 0.6482 & 0.6345 & 0.6315 & 0.6321 \\
0.90 & 0.5824 & 0.5791 & 0.5789 & 0.5786 & 0.5785 & 0.5783 \\
1.00 & 0.5037 & 0.4981 & 0.4925 & 0.4911 & 0.4901 & 0.4906 \\
1.20 & 0.3476 & 0.3403 & 0.3391 & 0.3379 & 0.3377 & 0.3381 \\
\hline
\end{tabular}

${ }^{*}$ Corresponds to the results of Wang (1986)

\section{Conclusion}

The work is motivated by the need for an analytical solution to the evaluation of the hydrodynamic coefficients and loads on a submerged sphere in waves. The influence of body submergence and water depth is demonstrated through results in tabular form. Many of the numerical methods depend on the summation of wave pressure on the wetted body surface. Truncation of the complex matrix equation (25) by taking $N_{p}=20$ saves a lot of time. Consideration of multipole expansions has, on one hand taken care of the singularities in the flow, and on the other hand, help in finding a suitable expression for the diffraction potential. This potential gives us the description of the flow as well helps us in determining the exciting forces. It is

Table 2. Heave exciting forces $f_{z d}$ for $(h / a=1 \cdot 25)$.

\begin{tabular}{lcccccc}
\hline & \multicolumn{7}{c}{$d / a$} \\
\cline { 2 - 7 }$K a$ & 2.5 & 3.0 & $5 \cdot 0$ & $11 \cdot 0$ & $20 \cdot 0^{*}$ & Wu \\
\hline $0 \cdot 1$ & 0.8241 & 0.9582 & 1.2041 & 1.3979 & 1.4671 & 1.4676 \\
$0 \cdot 20$ & 0.7965 & 0.9297 & 1.1505 & 1.3192 & 1.3294 & 1.3293 \\
$0 \cdot 30$ & 0.7752 & 0.9042 & 1.1421 & 1.2547 & 1.2609 & 1.2613 \\
$0 \cdot 40$ & 0.7598 & 0.8847 & 1.1167 & 1.1147 & 1.1162 & 1.1165 \\
0.50 & 0.7421 & 0.8624 & 0.9917 & 0.9867 & 0.9872 & 0.9870 \\
$0 \cdot 60$ & 0.7134 & 0.8261 & 0.9256 & 0.9269 & 0.9283 & 0.9288 \\
$0 \cdot 70$ & 0.6790 & 0.7931 & 0.8291 & 0.8304 & 0.8317 & 0.8321 \\
$0 \cdot 80$ & 0.6224 & 0.7391 & 0.7398 & 0.7404 & 0.7409 & 0.7411 \\
0.90 & 0.5631 & 0.6112 & 0.6123 & 0.6136 & 0.6149 & 0.6151 \\
1.00 & 0.4832 & 0.4841 & 0.4846 & 0.4850 & 0.4852 & 0.4854 \\
$1 \cdot 10$ & 0.4162 & 0.4221 & 0.4247 & 0.4261 & 0.4275 & 0.4274 \\
1.20 & 0.3281 & 0.3289 & 0.3286 & 0.3284 & 0.3283 & 0.3284 \\
\hline
\end{tabular}

${ }^{*}$ Corresponds to the results of Wang (1986) 
Water-wave diffraction by spherical structures

Table 3. Surge exciting forces $f_{x d}$ for $(d / a=6)$.

\begin{tabular}{|c|c|c|c|c|}
\hline \multirow[b]{2}{*}{$K a$} & \multicolumn{4}{|c|}{$h / a$} \\
\hline & $1 \cdot 25$ & 1.75 & $3 \cdot 00$ & $5 \cdot 0^{*}$ \\
\hline $0 \cdot 1$ & $2 \cdot 0117$ & $1 \cdot 8694$ & 1.7021 & 1.7023 \\
\hline 0.2 & $1 \cdot 5106$ & $1 \cdot 2864$ & 0.9462 & 0.9464 \\
\hline $0 \cdot 3$ & $1 \cdot 2461$ & 0.9862 & 0.6741 & 0.6744 \\
\hline 0.4 & 1.0967 & 0.7421 & $0 \cdot 3909$ & 0.3911 \\
\hline 0.5 & 0.8984 & 0.6842 & $0 \cdot 3646$ & 0.3648 \\
\hline 0.6 & 0.7791 & 0.5098 & $0 \cdot 2517$ & 0.2518 \\
\hline 0.7 & 0.7364 & 0.4726 & $0 \cdot 2021$ & 0.2022 \\
\hline 0.8 & 0.6274 & $0 \cdot 3622$ & $0 \cdot 1271$ & 0.1273 \\
\hline 0.9 & 0.5097 & 0.2671 & 0.0983 & 0.0985 \\
\hline 1.0 & 0.4892 & 0.2491 & 0.0608 & 0.0607 \\
\hline $1 \cdot 2$ & 0.3972 & $0 \cdot 1977$ & 0.0323 & 0.0324 \\
\hline $1 \cdot 4$ & $0 \cdot 2947$ & $0 \cdot 1389$ & 0.0086 & 0.0086 \\
\hline 1.6 & 0.2566 & $0 \cdot 1082$ & 0.0016 & 0.0016 \\
\hline 1.8 & 0.2314 & 0.0627 & 0.0009 & 0.0009 \\
\hline $2 \cdot 0$ & $0 \cdot 2155$ & $0 \cdot 0419$ & 0.0005 & 0.0005 \\
\hline $2 \cdot 4$ & 0.1863 & 0.0219 & 0.0000 & 0.0000 \\
\hline $2 \cdot 8$ & $0 \cdot 1456$ & 0.0098 & 0.0000 & 0.0000 \\
\hline $3 \cdot 0$ & $0 \cdot 1178$ & 0.0039 & 0.0000 & 0.0000 \\
\hline
\end{tabular}

*Corresponds to the results of Wang (1986)

Table 4. Heave exciting forces $f_{z d}$ for $(d / a=6)$.

\begin{tabular}{|c|c|c|c|c|}
\hline \multirow[b]{2}{*}{$K a$} & \multicolumn{4}{|c|}{$h / a$} \\
\hline & $1 \cdot 25$ & 1.75 & $3 \cdot 00$ & $5 \cdot 0^{*}$ \\
\hline $0 \cdot 1$ & $1 \cdot 2561$ & 1.0692 & 0.6841 & 0.6843 \\
\hline $0 \cdot 2$ & $1 \cdot 2293$ & 0.9542 & 0.6194 & 0.6196 \\
\hline $0 \cdot 3$ & $1 \cdot 2007$ & 0.7781 & 0.4382 & 0.4383 \\
\hline 0.4 & $1 \cdot 1467$ & 0.7392 & 0.3922 & 0.3924 \\
\hline $0 \cdot 5$ & 0.9724 & 0.6107 & $0 \cdot 2965$ & 0.2966 \\
\hline $0 \cdot 6$ & $0 \cdot 8862$ & 0.5566 & $0 \cdot 2264$ & 0.2265 \\
\hline 0.7 & 0.6833 & 0.4192 & 0.1791 & 0.1792 \\
\hline $0 \cdot 8$ & 0.6374 & 0.3643 & 0.1267 & $0 \cdot 1268$ \\
\hline $0 \cdot 9$ & 0.5277 & $0 \cdot 2818$ & $0 \cdot 1082$ & $0 \cdot 1084$ \\
\hline $1 \cdot 0$ & 0.4721 & 0.2364 & 0.0927 & 0.0927 \\
\hline 1.4 & $0 \cdot 2021$ & $0 \cdot 1028$ & 0.0237 & 0.0237 \\
\hline $1 \cdot 8$ & $0 \cdot 1161$ & $0 \cdot 0711$ & $0 \cdot 0081$ & $0 \cdot 0081$ \\
\hline $2 \cdot 0$ & $0 \cdot 0986$ & 0.0529 & $0 \cdot 0072$ & 0.0072 \\
\hline $2 \cdot 4$ & $0 \cdot 0583$ & 0.0294 & 0.0039 & 0.0039 \\
\hline $2 \cdot 8$ & 0.0185 & 0.0129 & 0.0014 & $0 \cdot 0014$ \\
\hline $3 \cdot 0$ & $0 \cdot 0011$ & $0 \cdot 0081$ & 0.0005 & 0.0005 \\
\hline
\end{tabular}

*Corresponds to the results of Wang (1986) 
considered advantageous to provide analytical solutions to the exciting forces as a basis for validating numerical results when applied to a body of spherical shape. Our method can be used to solve the radiation problem also wherein we can evaluate the related hydrodynamic coefficients such as added mass and damping coefficients. Furthermore, there is also scope for extending this result for a pair of spheres having the same radius. Consideration of nonlinear theory will be another positive step to examine whether neglecting the nonlinearities has any significant deviation from the present result. It will be worthwhile to consider the nonlinear theory and compare the results with the present one.

\section{References}

Bora S N 2002 Exciting forces due to diffraction of water waves on a sphere in finite depth water. WSEAS Trans. Math. 1: 180-185

Havelock T H 1931 The wave resistance of a spheroid. Proc. R. Soc. London A131: 275-284

Havelock T H 1955 Waves due to a floating sphere making periodic heaving oscillations. Proc. $R$. Soc. London A231: 1-7

Hulme A 1982 The wave forces acting on a floating hemisphere undergoing forced periodic oscillations. J. Fluid Mech. 121: 443-463

McLachlan N W 1941 Bessel functions for engineers (Oxford: University Press)

Thorne R C 1953 Multipole expansions in the theory of surface waves. Proc. Cambr. Philos. Soc. 49: $707-716$

Wang S 1986 Motions of a spherical submarine in waves. Ocean Eng. 13: 249-271

Wu G X 1994 Hydrodynamic forces on a submerged sphere undergoing large amplitude motion. $J$. Ship Res. 38: 272-277

Wu G X, Taylor E R 1987 The exciting force on a submerged spheroid in regular waves. J. Fluid Mech. 182: 411-426

Wu G X, Taylor E R 1988 Radiation and diffraction of water waves by a submerged sphere at forward speed. Proc. R. Soc. London A417: 433-461

Wu G X, Taylor E R 1989 On the radiation and diffraction of surface waves by submerged spheroids. J. Ship Res. 33: 84-92

Wu G X, Taylor E R 1990 The hydrodynamic forces on a submerged sphere moving in a circular path. Proc. R. Soc. London A428: 215-227

Wu G X, Witz J A, Ma Q, Brown D T 1994 Analysis of wave induced forces acting on a submerged sphere in finite water depth. Appl. Ocean Res. 16: 353-361 